\title{
Omeprazole Ameliorates Aspirin-Induced Gastroduodenal Injury
}

\author{
JAMES M. SCHEIMAN, MD, ELIZABETH M. BEHLER, RN, KATHRYN M. LOEFFLER, BS, \\ and GRACE H. ELTA, MD
}

\begin{abstract}
Aspirin and nonsteroidal antiinflammatory drugs (NSAIDs) damage the gastroduodenal epithelium by two mechanisms: direct toxic effects and effects related to the depletion of endogenous prostaglandins. The prostaglandin-depleted mucosa has increased suceptibility to luminal aggressive factors, yet the role of acid in the pathogenesis of the NSAID ulcer is controversial. In humans, standard doses of $\mathrm{H}_{2}$-receptor antagonists prevent only duodenal injury and provide no protection for the gastric mucosa. It is not known whether more potent suppression of acid can prevent NSAID damage. Twenty healthy volunteers were randomized to a double-blind, placebo-controlled, crossover study to determine if omeprazole, $40 \mathrm{mg} /$ day prevents gastroduodenal injury due to two weeks of aspirin administration (650 mg four times a day). The severity of mucosal injury was quantitated by endoscopy and stratified by a scale from 0 (normal) to 4 (ulcer). Fourteen of the 20 subjects had less gastric injury during cotherapy with omeprazole. All six with no difference received aspirin plus omeprazole in the first treatment period. Omeprazole significantly decreased aspirin-induced gastric mucosal injury $(\mathrm{P}<0.001$, Wilcoxon signed-rank test). Omeprazole protected $85 \%$ of subjects from extensive gastric erosions (often associated with evidence of intraluminal bleeding) or ulceration, whereas $70 \%$ of the subjects developed aspirin-induced grades 3 and 4 gastric injury on placebo $(\mathrm{P}<0.01$ by $\chi^{2}$ ). No subject taking omeprazole developed duodenal injury of any grade, while $50 \%$ taking placebo developed erosions and $15 \%$ had ulcer $(\mathrm{P}<0.001)$. Medication side effects were mild in the majority of subjects. Heartburn occurred in seven subjects on aspirin and placebo vs one on aspirin and omeprazole $(\mathrm{P}<0.01)$. Salicylate levels were $7.39 \pm 4.72$ $\mathrm{mg} / \mathrm{dl}(535 \pm 340$ umolliter $)$ in the placebo group and $6.95 \pm 4.3 \mathrm{mg} / \mathrm{dl}(503 \pm 311$ umol/liter) in the omeprazole group. We conclude that omeprazole, $40 \mathrm{mg} /$ day eliminates duodenal injury and markedly ameliorates gastric injury due to administration of aspirin $2600 \mathrm{mg} /$ day. Omeprazole prophylaxis of NSAID injury deserves further study.
\end{abstract}

KEY WORDS: nonsteroidal antiinflammatory drugs; peptic ulcer disease; acid secretion.

Manuscript received September 28, 1992; revised manuscript received January 15, 1993; accepted March 15, 1993.

From the Division of Gastroenterology, Department of Internal Medicine, University of Michigan Medical School, Ann Arbor, Michigan.

Supported in part by NIH grant MO1 RR00042 and a gift from Merck Sharpe and Dohme Research Laboratories. The pharmaceutical company had no input into the study design, analysis of results, or review of the manuscript prior to publication.

Address for reprint requests: Dr. James M. Scheiman, GI Section (111D), VA Medical Center, 2215 Fuller Road, Ann Arbor, Michigan 48105.
Aspirin and other nonsteroidal antiinflammatory drugs (NSAIDs) are a very common cause of macroscopic gastric and duodenal mucosal injury. Epidemiologic estimates of NSAID usage suggest that up to $1.2 \%$ of the United States population use these agents daily (1). Given the vast number of patients exposed to NSAIDs, their toxicity to the upper gastrointestinal tract probably represents the most frequent drug side effect in the United States 
(2). NSAIDs initiate mucosal injury, culminating in ulceration, and promote complications such as bleeding and perforation (3).

Prospective endoscopic studies of rheumatic patients receiving chronic aspirin therapy have confirmed a high incidence of both gastric and duodenal damage $(4,5)$. Gastric ulcers were noted in $17 \%$, erosions in $40 \%$, with duodenal ulcers and erosions seen in $4 \%$ and $13 \%$, respectively. Additionally, these studies show that NSAID-related gastroduodenal injury correlates poorly with symptoms. The absence of symptoms in the presence of NSAID injury has ominous potential. NSAID use has been implicated as an important risk factor for the development of complicated ulcer disease requiring surgery (6) or causing death (7).

NSAIDs are thought to cause mucosal injury via two mechanisms. The first is by direct local toxicity to mucosa. The second effect is systemically mediated by cyclooxygenase inhibition, which depletes endogenous prostaglandin levels important in the maintenance of mucosal integrity. NSAID-induced injury in animal models is ameliorated, although not prevented by antisecretory agents, suggesting that the presence of acid may be required for either the direct or systemic toxicity to occur. In a recent study utilizing rabbits rendered prostaglandin deficient with antiprostaglandin antibodies, the maintenance of intragastric $\mathrm{pH}>5$ with omeprazole markedly reduced gastric ulcer formation (8). Whether the dictum "no acid-no ulcer" is true for NSAIDassociated ulcers in humans remains unclear. While $\mathrm{H}_{2}$-receptor antagonists have protected against short-term ( $\leq 1$ week) NSAID administration in normal volunteers (9), ranitidine protected only the duodenum from NSAID injury in longer-term studies in rheumatic patients (10). There is recent evidence that cotherapy with omeprazole results in a dose-dependent reduction in gastric mucosal blood loss caused by short-term (two-day) aspirin use (11). High-dose omeprazole (40 mg twice a day), which results in almost complete acid suppression, reduced mucosal blood loss to a level similar to that obtained with placebo.

To further investigate the importance of acid in NSAID-induced damage, we studied the effect of potent acid suppression with the proton pump inhibitor omeprazole on long-term (two week) aspirin-induced injury in normal volunteers. Gastroduodenal damage was quantitated endoscopically.
Table 1. Endoscopic Rating Scale

\begin{tabular}{cl}
\hline Grade & Endoscopic appearance \\
\hline 0 & normal (erythema included) \\
1 & $\leq 5$ erosions \\
2 & $6-15$ erosions \\
3 & $>15$ erosions, and/or \\
4 & spontaneous bleeding \\
\hline
\end{tabular}

\section{MATERIALS AND METHODS}

Twenty healthy volunteers between the ages of 18 and 65 were recruited by advertisement. Criteria for subject selection included no history of significant medical problems, no regular medication use, no history of gastrointestinal disease, no allergy to aspirin, no alcohol abuse, and the absence of recent or regular use of NSAIDs. The subjects were randomly assigned to a single capsule containing either omeprazole $40 \mathrm{mg} /$ day or identical placebo (Merck Sharp and Dohme, West Point, Pennsylvania), and aspirin $650 \mathrm{mg}$ (two 325-mg tablets, Geneva Generics, Broomfield, Colorado) four times per day for two weeks. A baseline endoscopy to ensure mucosal integrity was performed on all subjects prior to drug administration. On the 15th study day, endoscopy was performed to assess gastroduodenal damage. All endoscopies were done with local oropharyngeal anesthesia (Lidocaine, Astra) and intravenous sedation (Versed, Roche) after an overnight fast. Both the subjects and endoscopists (J. Scheiman, G. Elta) were blinded to the cotherapy, omeprazole versus placebo, with aspirin. Prior to endoscopy, serum was obtained for measurement of salicylate levels reflecting blood concentrations approximately $10 \mathrm{hr}$ after the preceding dose of aspirin.

After a two-week period to "wash out" the drug, a second baseline endoscopy was performed to ensure mucosal integrity. Subjects were then crossed over to either omeprazole or placebo plus aspirin for an additional two weeks. To ensure healing of prior injury, a few subjects with severe damage were given a brief course of antacid therapy ( $30 \mathrm{cc}$ four times a day for five days) during the wash-out period. Medication compliance was confirmed by pill count of returned medication at the end of each treatment period. The protocol and consent form for this study were reviewed and approved by the Human Use Committee, Ann Arbor, Michigan.

Endoscopic Scoring System. Endoscopic injury was quantitated by the endoscopic rating scale described in Table 1. An erosion was defined as a white-based lesion $<4 \mathrm{~mm}$ in length surrounded by erythematous mucosa. Ulcers were defined as circumscribed breaks in the mucosa with appreciable depth $\geq 4 \mathrm{~mm}$ in size. The presence of fresh or old blood in the stomach or duodenum was also noted. Subjects kept a diary to report the symptoms of abdominal pain, heartburn, nausea, vomiting, tarry stool, skin rash, tinnitus, diarrhea, and headache. The intensity was rated as mild, moderate, severe, and intolerable on a 1-4 scale. Subjects completed a baseline symptom score reflecting these symptoms in the previous year and also during the two-week period off all medications. 
Table 2. Posttreatment Gastric Mucosal Scores

\begin{tabular}{lcccccc}
\hline & \multicolumn{6}{c}{ Score } \\
\cline { 2 - 7 } \multicolumn{1}{c}{ Treatment } & 0 & 1 & 2 & 3 & 4 & Total \\
\hline Placebo & 0 & 1 & 5 & 9 & 5 & 20 \\
Omeprazole 40 mg/day & 8 & 3 & 6 & 2 & 1 & 20 \\
\hline
\end{tabular}

Statistical Methods. The use of a double-blind, crossover design allows analysis of the distribution of the paired injury scores in the omeprazole and placebo groups by the Wilcoxcon signed-rank test. For the purposes of the study, clinically significant protection by omeprazole was defined as a score of $\leq 1$ for the gastric mucosa and 0 for duodenal mucosa. The efficacy for omeprazole protection was analyzed using $\chi^{2}$ test with Yates' correction. Salicylate levels were compared by the Student's $t$ test for paired data.

\section{RESULTS}

Twenty-six subjects entered the study and 20 subjects completed it. The 20 volunteers included 11 men and 9 women with an age range of 18-44 years (mean \pm SD was $27 \pm 6$ years). Only two female subjects $(10 \%)$ smoked cigarettes, smoking 4-10 cigarettes per day. Ethanol use was not proscribed for this study. The distribution of ethanol use during the study was: $40 \%$, none; $15 \%, 1$ drink/ week; 30\%, 2-3 drinks/week; 15\%, 8-20 drinks/ week. Two subjects were dropped due to baseline erosion on entry endoscopy. Two subjects were dropped due to medication noncompliance (aspirin), and one for personal reasons. One subject in the omeprazole arm dropped due to rash and facial puffiness. Analysis of returned medications indicated that $90 \%$ of the subjects took all medications prescribed; two subjects missed one to three days of medications.
Table 3. Gastric Therapeutic Success Rate*

\begin{tabular}{lccc}
\hline \multicolumn{1}{c}{ Treatment } & Protection & No Protection & Total \\
\hline Placebo & $2(10 \%)$ & 18 & 20 \\
Omeprazole $40 \mathrm{mg} /$ day & $11(55 \%) \dagger$ & 9 & 20 \\
\hline
\end{tabular}

*Score of $\leq 1=$ protection.

$\dagger P<0.01$ by $\chi^{2}$.

Prevention of Gastric Lesions. The distribution of posttreatment gastric endoscopic scores is shown in Table 2. A plot of individual gastric scores for each subject is shown in Figure 1. Fourteen of 20 subjects had less injury on omeprazole $40 \mathrm{mg} /$ day vs placebo. There were six subjects who had no difference in gastric injury, all received ASA and omeprazole in the initial treatment period before crossover. No subject had greater injury on omeprazole. Omeprazole significantly decreased gastric mucosal injury score due to aspirin, $650 \mathrm{mg}$ four times a day, compared to placebo $(P<0.001)$.

Table 3 summarizes the therapeutic success rate for gastric protection by omeprazole. Applying a rigorous definition to therapeutic success with a score $\leq 1$ ( $\leq 5$ erosions), omeprazole significantly protected the gastric mucosa in $55 \%$ vs $10 \%$ of subjects $(P<0.01)$. Aspirin induced extensive erosive injury, which was frequently associated with endoscopic evidence of intraluminal bleeding or ulceration (grades 3 and 4 injury) in $70 \%$ of placebo subjects compared to $15 \%$ of subjects on omeprazole cotherapy $(P<0.001)$.

Table 4 summarizes the final duodenal endoscopic score for the subjects. No subject taking omeprazole developed aspirin-induced duodenal injury, a therapeutic success rate of $100 \%$. Fifty percent of subjects on placebo developed duodenal
A

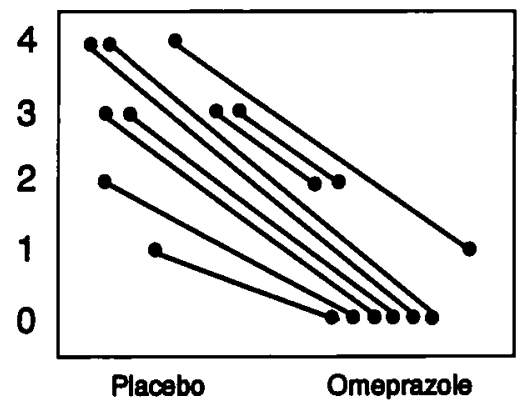

B

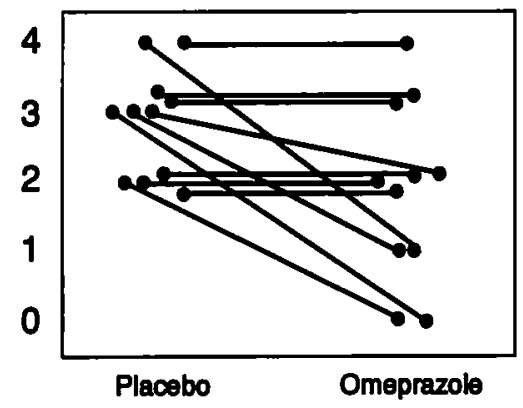

Fig 1. Final gastric injury scores for the 20 subjects. (A) Subjects receiving aspirin + placebo initially. (B) Subjects receiving aspirin + omeprazole initially. Analysis of all subjects demonstrates omeprazole significantly decreases gastric injury $(P<0.001$, Wilcoxon signed-rank test). 
Table 4. Postrteatment Duodenal Mucosal Scores

\begin{tabular}{lcccccc}
\hline & \multicolumn{7}{c}{ Score } \\
\cline { 2 - 7 } \multicolumn{1}{c}{ Treatment } & 0 & 1 & 2 & 3 & 4 & Total \\
\hline Placebo & 10 & 4 & 2 & 1 & 3 & 20 \\
Omeprazole $40 \mathrm{mg} /$ day & 20 & 0 & 0 & 0 & 0 & 20 \\
\hline
\end{tabular}

erosions, and $15 \%$ developed frank ulceration $(P<$ 0.01 , Wilcoxon signed rank test).

Adverse Symptoms. Medication side effects were mild in the majority of subjects. One subject was dropped from the protocol due to a possible drug reaction with skin rash and facial puffiness while taking aspirin and omeprazole. Tinnitus was noted in three subjects, prompting a reduction in aspirin dose to three times a day in one subject taking placebo, with resolution of symptoms. Two subjects noted nausea, one taking placebo. Abdominal pain occurred in five subjects on placebo and in four on omeprazole; pain intensity was similar in both groups. Heartburn was the most frequent complaint, which occurred in seven subjects taking aspirin and placebo vs one subject taking cotherapy with omeprazole, a significant difference $(P \leq 0.01$ by $\left.\chi^{2}\right)$. There was no correlation between symptoms and' endoscopic scores. The remainder of reported symptoms were rare and of equal frequency prior to and off all medications versus during treatment.

Salicylate Levels. Salicylate levels were $7.39 \pm$ $4.72 \mathrm{mg} / \mathrm{dl}(535 \pm 340 \mu \mathrm{mol} / \mathrm{liter})$ in the placebo group and $6.95 \pm 4.28 \mathrm{mg} / \mathrm{dl}(503 \pm 311 \mu \mathrm{mol} /$ liter $)$ in the omeprazole group. There was no significant difference in salicylate levels in the two groups or any correlation between salicylate level and endoscopic injury score.

\section{DISCUSSION}

Strategies to prevent injury from aspirin and other NSAIDs have evolved from our understanding of the combination of local and systemic mechanisms of mucosal injury (12). To avoid local toxicity, enteric coating and prodrugs that bypass gastric absorption have been utilized. Although mucosal damage appears diminished by this strategy, the systemically mediated depletion of gastroduodenal prostaglandins still makes these agents ulcerogenic. It has been suggested that NSAIDinduced inhibition of prostaglandin synthesis is unlikely to cause damage per se, but causes the mucosa to be more susceptible to injury by acid, bile salts, or ethanol (13). Since prostaglandin de- pletion is fundamental to the pathogenesis of the NSAID ulcer, prophylactic replacement of exogenous prostaglandins is logical (14). The oral $\mathrm{PGE}_{1}$ analog misoprostol is currently the only agent proven effective in the prevention of gastric ulcers in patients taking NSAIDs. However, drug-induced diarrhea and abdominal discomfort limit both physician and patient acceptance of misoprostol. Diarrhea occurs in up to $39 \%$ of patients taking misoprostol, although lower doses $(100 \mu \mathrm{g}$ four times a day) are better tolerated. These side effects may lead to rates of drug discontinuation above the 5\% reported in clinical trials (3). Given the limitations in patient and physician acceptance of oral prostaglandins, a search for other prophylactic strategies for NSAID ulceration seems worthwhile.

The efficacy of traditional doses of $\mathrm{H}_{2}$-receptor antagonists for healing of NSAID-induced ulcers despite continued NSAID use is controversial. Recently, a large multicenter study showed profound acid inhibition using high-dose ( $40 \mathrm{mg} /$ day) omeprazole was effective in the treatment of NSAIDassociated gastric ulcers (15). The healing and relapse rate of three treatment regimens for benign gastric ulcer were compared: $40 \mathrm{mg}$ omeprazole every day, $20 \mathrm{mg}$ omeprazole every day, and 150 $\mathrm{mg}$ ranitidine twice a day. Analysis of the subgroup with NSAID-associated ulcers who continued to receive concurrent NSAIDs showed four-week healing rates of $81 \%$ in the $40 \mathrm{mg} /$ day omeprazole group, $61 \%$ in the $20 \mathrm{mg} /$ day group, and $32 \%$ in those treated with ranitidine. At eight weeks, the percentages healed were 95,82 , and $53 \%$. There was also significantly less relapse of both symptoms and ulceration in the omeprazole-treated groups at six-month follow-up off all medication. The ability to heal NSAID ulcers with acid suppression while NSAIDs are continued suggests that NSAIDinduced ulcers depend on acid-peptic activity. Perhaps the prostaglandin-depleted mucosa is so sensitive to acid injury that more acid inhibition is required than obtained with $\mathrm{H}_{2}$-receptor antagonists both for healing of established ulcers despite continued NSAID use and for prophylaxis of ulcer formation.

We studied normal volunteers to determine the efficacy of omeprazole $40 \mathrm{mg} /$ day for the prevention of chronic (two-week) gastroduodenal damage from $2600 \mathrm{mg} /$ day of aspirin. It is controversial whether volunteer studies can predict efficacy of therapies designed to prevent NSAID ulceration. However, it has been observed that NSAID doses that produce 
significant injury in volunteers tend to cause similar injury in patients (16). The advantages of volunteer studies are that they are faster to complete and potentially safer than those in patients. The normal volunteer studies comparing the efficacy of misoprostol vs $\mathrm{H}_{2}$-receptor antagonists for the prevention of NSAID injury had similar results to the subsequent studies in arthritic patients. Unfortunately, lack of injury in short-term studies has not always correlated with a lack of injury in patients on longer-term therapy $(9,10)$. Aspirin-induced injury decreases despite continued administration, an effect known as "gastric adaptation" (17). This reduction in injury begins immediately after aspirin administration and occurs in two to eight weeks (median 4.5 weeks) in subjects taking $650 \mathrm{mg}$ four times a day (18). The somewhat intermediate two week treatment period in this study was chosen to allow volunteer compliance.

Aspirin inhibits cyclooxygenase, depletes endogeneous prostaglandins, and produces the most dramatic acute topical injury of the NSAIDs. The dissociation constant of aspirin is 3.5 ; typical NSAIDs have ionization constraints in the 3-5 range. In the presence of acid, these drugs are nonionized and freely diffuse across cell membranes. Once inside the cell, the elevated intracellular $\mathrm{pH}$ promotes dissociation to the ionized form. Once ionized, aspirin remains water soluble and trapped intracellularly. Because the nonionized form remains in equilibrium across the cell membrane, the total intracellular drug concentration (ionized and nonionized) will be much higher than outside the cell, an effect known as ion trapping (19). Ion trapping allows direct cellular injury due to the toxicity of high intracellular levels of salicylate. These toxic effects lead to abnormal ion flux across the epithelium, with increased $\mathrm{H}^{+}$backdiffusion occurring rapidly. These changes in membrane permeability may be secondary to impairment of cellular energy metabolism (13). Other NSAIDs appear to damage mucosa through similar mechanisms, although quantitative levels of topical injury among NSAIDs vary considerably. Extrapolation of our data regarding the ability of omeprazole to ameliorate injury caused by NSAIDs other than aspirin requires experimental confirmation.

Acid suppression may decrease topical injury to aspirin by raising intragastric $\mathrm{pH}$ to maintain salicylate in its ionized form. Alternatively, acid suppression may decrease local damage by simply decreasing hydrogen ion back diffusion. The ability of high-dose (40 mg twice a day) omeprazole therapy to abolish short-term aspirin-induced $(900 \mathrm{mg}$ twice a day for two days) gastric blood loss suggests that the presence of luminal acid is important for initiation of NSAID ulceration. Omeprazole $20 \mathrm{mg}$ /day in the same protocol significantly prevented aspirininduced blood loss but was less effective than the higher dose (11). Long-term use of omeprazole at 80 $\mathrm{mg} /$ day is currently impractical, prompting us to choose $40 \mathrm{mg} /$ day.

Omeprazole $40 \mathrm{mg} /$ day significantly prevented both gastric and duodenal injury due to $2600 \mathrm{mg}$ aspirin/day over the two-week period of our study. Since our endoscopic score is arbitrary, the definition of therapeutic efficacy is a matter of judgment. Omeprazole $40 \mathrm{mg} /$ day prevented $95 \%$ of subjects from developing ulceration, $85 \%$ from having $>15$ erosions (all $\leq 3 \mathrm{~mm}$ in size), and $55 \%$ from having $>5$ erosions. In the subjects given placebo, $25 \%$ developed gastric ulcers, $70 \%$ had grade 3 injury or worse, and all $95 \%$ had at least grade 2 injury. Omeprazole was $100 \%$ effective in preventing duodenal injury. Skeptics question the importance of preventing erosions in the absence of direct evidence linking erosions to chronic ulcers. However, in a recent trial comparing the efficacy of misoprostol vs sucralfate for the prevention of NSAID ulcers, the presence of erosions upon study entry was associated with an increased risk of gastric ulcer (20), suggesting that erosions may develop into chronic ulcers with continued NSAID exposure. A study of longer duration in chronic NSAID users is necessary to determine if the observed decrease in aspirin-induced gastric and duodenal injury by omeprazole cotherapy reduces the number of chronic ulcers and their associated complications.

Omeprazole $40 \mathrm{mg} / \mathrm{day}$, a dose that decreases basal acid output by $80-93 \%$ and decreases intragastric acidity by $92-94 \%$, markedly ameliorated gastric injury due to aspirin. This effect appears to have occurred independent of a major influence upon salicylate absorption, since serum levels were not significantly different in each arm of the study. Only a detailed pharmacokinetic study could ensure the absence of any effect of omeprazole on aspirin absorption. The continued presence of injury in humans during this degree of acid suppression suggests that virtual anacidity, as observed with abolition of aspirin microbleeding with $80 \mathrm{mg}$ omeprazole/day, is required to prevent all of the acute topical injury of aspirin. Since we did not quantitate acid secretion in our subjects and there is known 
interindividual variability in omeprazole's ability to inhibit acid secretion (21), it is possible that the six subjects who developed equivalent levels of damage on omeprazole and placebo had weaker acid suppression. Furthermore, since omeprazole serum levels were not measured, absolute compliance cannot be confirmed. An alternative explanation for the lack of gastroprotection in five subjects is gastric adaptation. All of the subjects receiving placebo and aspirin in the initial treatment period had reduced gastric injury on omeprazole, six by two grades and three by one grade. In contrast, all six subjects who developed identical injury on placebo and omeprazole received cotherapy with omeprazole initially, including the subject who developed ulceration. Although the two-week wash-out period was designed to allow wash-out of omeprazole (21), it is possible that adaptive processes stimulated by two weeks of aspirin therapy remained activated and prevented the even more severe damage that would have occurred with aspirin and placebo.

Adverse effects in this study were uncommon. Two subjects developed a rash on omeprazole. This prompted discontinuation of study participation in one subject, but without rechallenge a definite relationship to omeprazole is uncertain. The other subjects' rash resolved during continued treatment. All other side effects were nearly identical in both treatment phases. Omeprazole significantly ameliorated heartburn related to aspirin uptake, an effect not observed with misoprostol prophylactic therapy.

Is long-term therapy with omeprazole, in doses of 20-40 mg/day, a practical consideration? The drug is costly and long-term use has not been proven to be safe. Pronounced acid inhibition leads to hypergastrinemia and increases the risk of enterochromaffin-like cell hyperplasia with a theoretical increased risk of carcinoid tumor formation. Furthermore, prolonged hypochlorhydria can lead to bacterial overgrowth and increased nitrosamine levels and a theoretical increased risk of gastric cancer (21). However, patients with Zollinger-Ellison syndrome have received this drug long-term, and its adverse effect profile has been similar to placebo or $\mathrm{H}_{2}$ receptor antagonists (22). Additionally, omeprazole can induce cytochrome P-450 IA2 in hepatocytes (23) and IA1 in the proximal gastrointestinal epithelium (24), raising the question of increased potential for carcinogenesis. This speculation remains totally hypothetical (25). No evidence for genotoxicity of omeprazole has been observed in recently published investigations (26). NSAID injury leads to
70000 hospitalizations and 7000 deaths annually in the United States (27). The population at greatest risk for NSAID-induced damage is elderly, thus exposure to very long-term omeprazole may be limited. Further studies are needed to ascertain the efficacy of omeprazole prophylaxis of NSAID injury and determine if the benefits of this therapy outweigh its risk and cost.

\section{ACKNOWLEDGMENTS}

The authors would like to thank the outpatient nurses of the Clinical Research Center of the University Hospital for their assistance with the endoscopies, and Daniel Cutler, Medical Media Service at the Ann Arbor VAMC for his preparation of the figure.

\section{REFERENCES}

1. Baum C, Kennedy DL: Utilization of anti-inflammatory drugs. Arthritis Rheum 28:686-689, 1984

2. Fries JF, Miller SR, Spitz PW, Williams CA, Hubert HB, Bloch DA: Toward an epidemiology of gastropathy associated with nonsteroidal anti-inflammatory drug use. Gastroenterology 96:647-655, 1989

3. Soll AH, Weinstein WM, Kurata J, Williams CA, Hubert $\mathrm{HB}$, Bloch DA: Nonsteroidal antiinflammatory drugs and peptic ulcer disease. Ann Intern Med 114:307-319, 1991

4. Silvoso GR, Ivery KJ, Butt JH, Lockard OO, Holt SD, Sisk $\mathrm{C}$, Baskin WN, Mackercher PA, Hewett J: Incidence of gastric lesions in patients with rheumatic disease of chronic aspirin therapy. Ann Intern Med 91:517-520, 1979

5. Lockark OO, Ivey KJ, Butt JH, Silvoso GR, Sisk C, Holt S: The prevalence of duodenal lesions in patients with rheumatic disease on chronic aspirin therapy. Gastrointest Endosc 26:5-7, 1980

6. Armstrong CP, Blower AL: Non-steroidal anti-inflammatory drugs and life threatening complications of peptic ulceration. Gut 28:527-532, 1987

7. Armstrong $\mathrm{CP}$, Whitelaw S: Death from undiagnosed peptic ulcer complications: A continuing challenge. Br J Surg 75:1112-1114, 1988

8. Lee M, Aldred K, Lee E, Prince DM, Feldman M: Importance of gastric acid in gastric ulcer formation in rabbits with antibody-induced prostaglandin deficiency. Gastroenterology 103:1467-1474, 1992

9. Berkowitz JM, Adler SN, Sharp JT, Warner CW: Reduction of aspirin-induced gastroduodenal mucosal damage with ranitidine. J Clin Gastroenterol 8(3):377-380, 1986

10. Ehsanullah RSB, Page MC, Tildesley G, Wood JR: Prevention of gastroduodenal damage induced by non-steroidal anti-inflammatory drugs: Controlled trial of ranitidine. BMJ 297:1017-1021, 1988

11. Daneshmend TK, Stein AG, Bhaskar NK, Hawkey CJ: Abolition by omeprazole of aspirin induced gastric mucosal injury in man. Gut 31:514-519, 1990

12. Schoen RT, Vender RJ: Mechanisms of nonsteroidal antiinflammatory drug-induced gastric damage. Am J Med 86:449-458, 1989

13. Cohen MM: Mechanisms of injury to gastric mucosa by 


\section{OMEPRAZOLE AMELIORATES ASPIRIN-INDUCED INJURY}

non-steroidal anti-inflammatory drugs and the protective role of prostaglandin. In Prostaglandins and Leukotrienes in Gastrointestinal Disease. W Domschke, HC Dammann (eds). Berlin, Springer-Verlag, 1988, pp 148-151

14. Graham DY, Agrawal NM, Roth SH: Prevention of NSAIDinduced gastric ulcer with misoprostol: Multicenter, doubleblind, placebo-controlled trial. Lancet 2:1277-1280, 1988

15. Walan A, Bader JP, Classen M, Lamers CBHW, Piper DW, Rutgersson K, Eriksson S: Effect of omeprazole and ranitidine on ulcer healing and relapse rates in patients with benign gastric ulcer. N Engl J Med 320:69-75, 1989

16. Laine L, Lanza FL: NSAID-induced gastroduodenal injury: What's the score? Gastroenterology 101:555-557, 1991 (letter)

17. Graham DY, Smith JL, Dobbs SM: Gastric adaptation occurs with aspirin administration in man. Dig Dis Sci 28:1-6, 1983

18. Graham DY, Smith JL, Spjut HJ, et al: Gastric adaptation. Gastroenterology 95:327-333, 1988

19. Brooks PM, Day RO: Nonsteroidal antiinflammatory drugsdifferences and similarities. N Engl J Med 324:1716-1725, 1991

20. Agrawal NM, Roth S, Graham DY, White RH, Germain B, Brown JA, Stromatt SC: Misoprostol compared with sucralfate in the prevention of nonsteroidal anti-inflammatory drug-induced gastric ulcer. Ann Intern Med 115:195-200, 1991

21. Maton PN: Drug therapy: Omeprazole. N Engl J Med 324:965-975, 1991

22. Holt S, Howden CW: Omeprazole: Overview and opinion. Dig Dis Sci 36:385-393, 1991

23. Diaz D, Fabre I, Daujat M, Saint Aubert B, Bories P, Michel $\mathrm{H}$, Maurel P: Omeprazole is an aryl hydrocarbon-like inducer of human hepatic cytochrome P450. Gastroenterology 99:737-747, 1990

24. McDonnell WM, Scheiman JM, Traber PG: Induction of cytochrome P4501A genes (CYP1A) by omeprazole in the human alimentary tract. Gastroenterology 103:1509-1516, 1992

25. Andersson T, Bergstrand R, Cederberg C, Eriksson S, Lagerstrom P-O, Skanberg I: Omeprazole treatment does not affect the metabolism of caffeine. Gastroenterology 101:943947, 1991

26. Holt S, Zhao-Hua Z, Powers RE: Observations on a proposed measure of genotoxicity in rat gastric mucosa. Gastroenterology 101:650-656, 1991

27. Fries JF: NSAID gastropathy: The second most deadly rheumatic disease? Epidemiology and risk appraisal. J Rheumatol 18(suppl 28):6-10, 1991 